\title{
Hospital Characteristics Associated with Increased Conversion Rates Among Organ Donors
}

\section{in New England}

Arturo J. Rios-Diaz, MD; Olubode A. Olufajo, MD, MPH; Jill Stinebring, MBA, RN, BSN, CPTC; Samantha

Endicott , MPH, CPTC; Brandon T. McKown, MBA; David Metcalfe, LLB, MBChB; Cheryl K. Zogg, MSPH, MHS; Ali Salim, MD, FACS

1. Center for Surgery and Public Health, Department of Surgery, Brigham and Women's Hospital, Harvard Medical School \& Harvard T.H. Chan School of Public Health. Boston, MA.

2. New England Organ Bank. Waltham, MA.

3. Division of Trauma, Burns, and Surgical Critical Care, Department of Surgery, Brigham and Women's Hospital. Harvard Medical School Brigham. Boston, MA.

4. Kadoorie Centre for Critical Care Research, University of Oxford, John Radcliffe Hospital, Oxford, UK.

\section{Corresponding author:}

Arturo J. Rios-Diaz, MD

Center for Surgery and Public Health

Department of Surgery, Brigham \& Women's Hospital.

One Brigham Circle, 1620 Tremont St, Suite 4-020.

Boston, MA 02115, USA

ariosdiaz@gmail.com

Abstract presented at the Academic Surgical Congress 2016 Annual Meeting, Jacksonville, FL, on Februrary 2-4, 2016.

Short title:

Word count: 1,871

Keywords: 


\section{Abstract (250/250)}

Background: It is unknown whether hospital characteristics affect institutional performance with regard to organ donation. We sought to determine which hospital- and patient-level characteristics are associated with high organ donor conversion rates after brain death (DBD) in hospitals located in New England.

Methods: Data were extracted from the New England Organ Bank (2011-2014) and other administrative data sources. Hospitals with $<4$ potential DBD were excluded. Hospitals were stratified into high-conversion hospitals $(\mathrm{HCH}$; upper-tertile) and low-conversion hospitals (LCH; lower-tertile) according to conversion rates. Hospital- and patient- characteristics were explored using descriptive statistics, Fisher's exact tests for binary/categorical variables and Mann-Whitney U tests for non-normally-distributed continuous variables.

\section{Results:}

There were 564 potential DBD donors in 27 hospitals. Conversion rates differed between hospitals in different states $(\mathrm{p}<0.001)$. HCH were more likely to be small (median bed size 194 vs. 337; $\mathrm{p}=0.024$ ), non-teaching hospitals ( $40 \%$ vs. $88 \%$; $\mathrm{p}=0.025)$, non-trauma center $(30 \%$ vs. $77 \%$; $\mathrm{p}=0.040)$. Potential donors differed between $\mathrm{HCH}$ and $\mathrm{LCH}$ in race $(\mathrm{p}<0.01)$ and mechanism of injury/disease process $(\mathrm{p}<0.01)$. In every recorded case, failure to convert potential donors to DBD was due to lack of consent from next-of-kin.

\section{Conclusion:}

There is significant variation between hospitals in terms of organ donor conversion rates. This suggests that there is a pool of potential donors in large specialized hospitals that are not successfully converted to DBD. Further work is necessary to determine why consent rates are lower in large regional centers and to design interventions targeted at optimizing the number of DBDs in these hospitals. 


\section{Introduction}

There are 121,650 Americans currently waiting to undergo organ transplantation.[1] Even though 2015 hit high records with 30,973 transplants,[1] the gap between supply and demand is substantial. It is for this reason that the Organ Procurement and Transplantation Network (OPTN) has identified increasing the number of transplants as the first key goal in their strategic plan.[2] One of the ways to increase the pool of organs available for transplantation is to increase the number of donors after brain death (DBD).[3]

It is important to understand the characteristics of hospitals and patients involved in organ donation in order to inform the design of interventions to increase the available pool of donor organs. A small number of previous studies have explored factors associated with increased organ donation. For instance, it has been suggested that some geographical locations are more likely to have higher authorization rates than others.[4-7] Demographic characteristics have also been associated with variations in consent rates: racial/ethnic minorities and older potential donors are less likely to consent[8], despite the fact that minority patients are over-represented on transplantation waiting lists.[9] Moving beyond individual patient characteristics, there is also evidence that hospital- and system-level factors are associated with organ donation. It has previously been shown that early Organ Procurement Organization (OPO) involvement in the consent process, having organ donor councils within the hospital, and the presence of in-house organ donation coordinators are associated with increased donor numbers.[10-15]

The objective of the present study was to explore associations between hospital- and patient-level characteristics and donor conversion rates after DBD. We hypothesized that there would be systematic differences in institutional characteristics between hospitals with high-performance and low-performance in terms of conversion rates. 


\section{Methods}

Data sources

Patient-level data from 2011-2014 were extracted from the New England Organ Bank (NEOB), which is a federally designated, non-profit organ procurement organization (OPO) that coordinates organ and tissue donation in all New England states (Connecticut, Maine, Massachusetts, New Hampshire, Rhode Island and Vermont) and Bermuda. This organization works in collaboration with over 150 hospitals and serves 11 million people in the region. Their staff participate in multiple aspects of donation processes including consent, maintenance of medical support of potential donors, coordination of organ procurement, allocation of organs according to the national transplant waiting list and organization of organ transportation to transplant centers or tissue processors.[16]

Hospital-level data were extracted from three different sources: (1) the American Hospital Association (AHA) Annual Survey Database (2012), which is a comprehensive repository of over 6,300 hospitals in the United States and includes up to 1,000 fields of data on organizational structure, facility and services, and geographic indicators[17] (2) the Joint Commission website;[18] which is a not-for-profit organization recognized nationwide that accredits and certifies nearly 21,000 health care organizations and programs in the country; and (3) the NEOB. Hospital level-data and patient-level data were linked by matching hospital names and geographic location (city and state).

\section{Study population}


Hospitals in New England with at least one DBD/year during the study period were included. The main unit of analysis was set at the hospital-level for hospital characteristics analysis. For processes and patient characteristics analysis, the unit of analysis was individual patients.

\section{Patient characteristics, process measures and hospital characteristics}

Patient-level variables extracted from the NEOB included age, gender, race, (White, Black, Hispanic, other) and disease process or mechanism of injury. Process measures included consent rate, firstperson authorization rate (potential donors that were registered as donors), proportion of patients approached by OPO staff, timeliness of referral of potential donors, and reasons for declining consent (for those whose consent was not obtained). Hospital-level variables extracted included transplant center designation (from NEOB data), stroke center designation (from Joint Commission data), trauma center designation, hospital bed size, teaching status (defined as hosting an Accreditation Council for Graduate Medical Education [ACGME] accredited physician training program or as member of the Council of Teaching Hospitals [COTH]), Intensive Care Unit (ICU) services, trauma center self-designation, ownership (for-profit or non-for-profit), urbanicity (defined by Core Based Statistical Area codes corresponding to population $\geq 2.5$ million), and state location (from AHA data).

\section{Outcome measures}

The main outcome was donor conversion performance of individual hospitals. Conversion rates were calculated for each hospital (actual DBD donors/potential DBD donors), which were then stratified into high-conversion hospitals (upper tertile) and low-conversion hospitals (lower tertile). Hospitals and patients in the middle tertile were excluded.

\section{Statistical analysis}


Hospital- and patient- characteristics were explored using descriptive statistics (frequencies and proportions), Fisher's exact (binary/categorical variables) and Mann-Whitney U (non-normallydistributed continuous variables) tests with a two-sided significance threshold set at $p<0.05$. All statistical analyses were performed using Stata Statistical Software: Release 13.0. (College Station, TX). Density maps with superimposed pie charts were created to illustrate the number of hospitals included per state and the proportion corresponding to high- and low-conversion hospital groups. All geographic analyses were performed using Tableau 9.2.6 (Seattle, WA). The research protocol was approved by the Partners Human Research Committee.

\section{Results}

There were 564 (464 in low-conversion and 100 in high-conversion hospitals) patients identified as potential donors at 27 individual hospitals (17 low-conversion and 10 high-conversion) during the study period. All potential donors for whom consent was obtained were converted into donors, and so the consent (patients/next-of-kin consented among those who were eligible) and conversion (actual donors among those who were eligible) rates were equal. The consent/conversion rate was $85 \%$ in the high-conversion group and $55 \%$ in the low-conversion group $(\mathrm{p}<0.001)$.

\section{Patient characteristics and process measures}

Patients in high- and low-conversion-hospitals did not differ statistically in terms of age (overall median age 46 years old [Interquartile range-IQR-: 27-58]) or sex (overall 56\% male) (Table 1). High- and low-conversion-hospitals differed in the proportion of potential donors who were White (89 vs. $64 \%$; $p<0.001)$, as well as in the mechanism of injury/disease process $(\mathrm{p}<0.001)$. Even though consent rates were significantly different ( $85 \%$ vs. $53 \%$; $<<0.001)$, first-person consent was comparable between the two groups ( $46 \%$ vs. 38\%; $=0.131)$. In high-conversion hospitals, 
potential donors' families were more likely to be approached for consent by NEOB staff (71\% vs. $55 \% ; \mathrm{p}=0.017$ ) and were typically referred to the OPO in a timely manner ( $92 \mathrm{vs.} \mathrm{89 \% ;} \mathrm{p}=0.226$ ).

Since all potential donors were approached by the NEOB, in every case, failure to convert potential donors to DBD was due to lack of consent from next-of-kin for a range of reasons, including relatives not wanting to wait or not wanting surgery for their loved ones, cultural/religious concerns, and not being aware of the patient's wishes. However, these reasons for declining consent were comparable between groups $(\mathrm{p}=0.376)$ (Table 3).

\section{Hospital characteristics associated with high conversion rates}

The conversion rates varied between $53 \%$ in low-conversion to $85 \%$ in high conversion hospitals (Table 2). High-conversion-hospitals were more likely to be smaller (median bed size 194 vs. 337; $\mathrm{p}=0.024$ ), non-teaching hospitals ( $40 \%$ vs. $88 \%$; $\mathrm{p}=0.025)$, and non-trauma centers $(30 \%$ vs. $77 \%$; $p<0.05)$. They did not differ in terms of urbanicity ( $p=0.692)$, ownership $(p=0.618)$, ICU $(p>0.990)$, availability of burn services $(\mathrm{p}=0.257)$, stroke center status $(\mathrm{p}=0.360)$, or transplant center status $(p=0.057)$. The volume of potential DBD donors was also significantly lower in the high-conversion group (7 [5-14] vs. 17 [11-32]; p=0.034). There were significant differences in conversion rates between hospitals in different states $(\mathrm{p}=0.024)$ with the highest proportion of high-conversion hospitals in Massachusetts (Table 3).

\section{Discussion}

This study integrated multiple sources of data to compare hospital and patient characteristics associated with increased organ donation performance among DBDs in New England. It found that small, non-academic, non-trauma center hospitals with relatively low volumes of potential donors are more likely to be high-performers in terms of converting DBDs. It also found that there are 
intrinsic differences at the patient-level between high- and low-conversion hospitals. These findings highlight several areas for improvement in the transplantation system.

Large academic trauma centers provide high quality of care for severely injured patients.[19] Besides having a large number of resources and specialized personnel, these hospitals are also required to meet certain standards in order to increase and maximize the organ donation pool.[20] These include establishing a relationship with an OPO, developing protocols for notification of the OPO about potential DBDs, having a defined procedure for reviewing organ donation outcomes, and implementing protocols for the declaration of neurologic death.[21] However, a prior study found that there was no association between these requirements and increased organ donation.[20] The finding that large academic trauma centers perform worse in terms of DBD conversion than smaller hospitals is surprising. This is because high-volume hospitals with considerable experience of organ donation might be expected to have developed protocols and expertise that would have improved their DBD conversion rates. Such volume-outcome relationships have been shown across a range of other surgical and critical care settings.[22] However, it is feasible that DBD conversion is impaired in these hospitals, because of their organizational complexity and the higher volume of patients managed in large academic trauma centers. This is consistent with the recent finding that some processes may be impaired and clinical outcomes compromised in busy trauma centers.[23, 24]

An alternative explanation for our finding is that the apparently poor performance of large academic trauma centers was driven by differences in patient case mix. Although we did not find differences in age or gender, there were dissimilarities in race origin. We found that there were a higher proportion of Black and Hispanic patients in low-conversion hospitals, which has been associated with lower conversion rates relative to White patients.[8, 25] It is nevertheless still 
possible that the processes in large academic trauma centers might influence whether or not consent to organ donation was forthcoming.

There are a number of limitations to this study. First, the relatively small sample size in our hospital analyses $(n=28)$ precluded the ability to control for potential confounders such as race and mechanism of injury or disease process. However, the proportion of minorities in the region is low and therefore it is unlikely that this finding skewed entirely our results. Moreover, other factors that could have been confounders such as age[8] and first-person authorization were comparable between groups. Second, it is uncertain whether our findings are generalizable outside of New England. Nonetheless, this methodology could be used by other OPOs or on a larger scale to identify hospitals with lower performance and target appropriate interventions to improve potential donors conversion. Third, we were unable to obtain more granular data on the number of organs obtained per donor and so it is possible that hospitals in the low-conversion group actually have a higher overall organ yield. Future studies should incorporate individual metrics such as conversion rates, consent rates, and organs transplanted per donor, and authorization rates to permit comprehensive benchmarking of hospitals.

There is substantial variation in potential donor conversion rates between different hospitals. These results suggest that hospital characteristics and processes as well as patient characteristics play a role in hospitals' conversion rates. In particular, there is a pool of potential donors in large regional academic and trauma center hospitals that are not successfully converted to DBD. These results suggest that logistical factors or clinical decision-making may be involved. Further work is necessary to determine why consent rates are lower in large regional hospitals to optimize the number of DBD. 


\section{Acknowledgement}

We would like to thank Andrea Suarez, MS, for providing assistance and support using Tableau for geospatial analyses. 


\section{References}

1. US Department of Health and Human Services - Health Resources and Services Administration. Organ Procurement and Transplantation Network 2016 [cited 2016 March 31]. Available from: https://optn.transplant.hrsa.gov/.

2. US Department of Health and Human Services - Health Resources and Services Administration. Organ Procurement and Transplantation Network Goal 12016 [cited 2016 April 1]. Available from: https://optn.transplant.hrsa.gov/governance/strategic-plan/goal$1 /$.

3. Goldberg DS, French B, Abt PL, Gilroy RK. Increasing the Number of Organ Transplants in the United States by Optimizing Donor Authorization Rates. American journal of transplantation : official journal of the American Society of Transplantation and the American Society of Transplant Surgeons. 2015;15(8):2117-25.

4. Delmonico FL, Sheehy E, Marks WH, Baliga P, McGowan JJ, Magee JC. Organ donation and utilization in the United States, 2004. American journal of transplantation : official journal of the American Society of Transplantation and the American Society of Transplant Surgeons. 2005;5(4 Pt 2):862-73.

5. Stogis S, Hirth RA, Strawderman RL, Banaszak-Holl J, Smith DG. Using a standardized donor ratio to assess the performance of organ procurement organizations. Health services research. 2002;37(5):1329-44.

6. Ojo AO, Pietroski RE, O'Connor K, McGowan JJ, Dickinson DM. Quantifying organ donation rates by donation service area. American journal of transplantation : official journal of the American Society of Transplantation and the American Society of Transplant Surgeons. 2005;5(4 Pt 2):958-66.

7. Ozcan YA, Begun JW, McKinney MM. Benchmarking organ procurement organizations: a national study. Health services research. 1999;34(4):855-74; discussion 75-8.

8. Goldberg D, Halpern S, Reese P. Deceased organ donation consent rates among racial and ethnic minorities and older potential donors. Crit Care Med. 2013;41:496-505.

9. Callender CO, Miles PV. Minority organ donation: the power of an educated community. Journal of the American College of Surgeons. 2010;210(5):708-15, 15-7.

10. Shirley S, Cutler J, Heymann C, Hart M. Narrowing the organ donation gap: hospital development methods that maximize hospital donation potential. The Journal of heart and lung transplantation : the official publication of the International Society for Heart Transplantation. 1994;13(5):817-23.

11. Miller LD, Gardiner SK, Gubler KD. Emergency department referral for organ donation: more organ donors and more organs per donor. American journal of surgery. 2014;207(5):728-33; discussion 33-4.

12. Sullivan H, Blakely D, Davis K. An in-house coordinator program to increase organ donation in public teaching hospitals. Journal of transplant coordination : official publication of the North American Transplant Coordinators Organization. 1998;8(1):40-2. 13. Salim A, Berry C, Ley EJ, Schulman D, Desai C, Navarro S, et al. In-house coordinator programs improve conversion rates for organ donation. The Journal of trauma. 2011;71(3):733-6. 
14. Salim A, Brown C, Inaba K, Mascarenhas A, Hadjizacharia P, Rhee P, et al. Improving consent rates for organ donation: the effect of an inhouse coordinator program. The Journal of trauma. 2007;62(6):1411-4; discussion 4-5.

15. Ehrle R. Timely referral of potential organ donors. Critical care nurse. 2006;26(2):88-93.

16. New England Organ Bank. New England Organ Bank Mission and Vision 2016 [cited 2016 April 3]. Available from: http://neob.org/mission-and-vision/.

17. American Hospital Association Health Forum. AHA Annual Survey Database ${ }^{\mathrm{TM}} 2016$ [cited 2016 March 30]. Available from: http://www.ahadataviewer.com/book-cdproducts/AHA-Survey/.

18. The Joint Commission. Stroke Certification Programs: Quality Check; 2016 [cited 2016 January 5]. Available from: http://www.qualitycheck.org/StrokeCertificationList.aspx.

19. MacKenzie EJ, Rivara FP, Jurkovich GJ, Nathens AB, Frey KP, Egleston BL, et al. A national evaluation of the effect of trauma-center care on mortality. The New England journal of medicine. 2006;354(4):366-78.

20. Malinoski DJ, Patel MS, Lush S, Willis ML, Navarro S, Schulman D, et al. Impact of compliance with the American College of Surgeons trauma center verification requirements on organ donation-related outcomes. Journal of the American College of Surgeons. 2012;215(2):186-92.

21. American College of Surgeons Committee on Trauma. Resources for optimal care of the injured patient. Chicago, IL: American College of Surgeons; 2014.

22. Nguyen YL, Wallace DJ, Yordanov Y, Trinquart L, Blomkvist J, Angus DC, et al. The Volume-Outcome Relationship in Critical Care: A Systematic Review and Meta-analysis. Chest. 2015;148(1):79-92.

23. Metcalfe D, Olufajo O, Rios-Diaz AJ, Haider A, Havens JM, Nitzschke S, et al. Are appendectomy outcomes in level I trauma centers as good as we think? The Journal of surgical research. 2016;202(2):239-45.

24. Metcalfe D, Olufajo OA, Zogg CK, Gates JD, Weaver MJ, Harris MB, et al. Are Older Adults With Hip Fractures Disadvantaged in Level 1 Trauma Centers? Medical care. 2016;54(6):616-22.

25. Davis BD, Norton HJ, Jacobs DG. The Organ Donation Breakthrough Collaborative: has it made a difference? American journal of surgery. 2013;205(4):381-6. 
Table 1. Patient characteristics stratified by DBD conversion rates per hospital.

\begin{tabular}{lccc}
\hline & $\begin{array}{c}\text { Low Conversion } \\
\text { (lowest tertile) } \\
n=464\end{array}$ & $\begin{array}{c}\text { High Conversion } \\
\text { (highest tertile) }\end{array}$ & $\boldsymbol{p}$ \\
Characteristic & $46(26-58)$ & $46(31-57)$ & 0.907 \\
\hline Age, median (IQR) & $(55.8)$ & $(56.0)$ & 0.974 \\
Male; (\%) & & & $<0.001$ \\
Race/ethnicity; (\%) & $(64.0)$ & $(89.0)$ & \\
White & $(13.6)$ & $(4.0)$ & \\
Black & $(15.3)$ & $(5.0)$ & \\
Hispanic & $(7.1)$ & $(2.0)$ & \\
Asian/Other & & $(23.0)$ & \\
Mechanism of injury or disease process; (\%) & $(43.3)$ & $(30.0)$ & \\
Intracranial hemorrhage/Stroke & $(22.2)$ & $(12.0)$ & \\
Cardiovascular; & $(17.0)$ & $(20.0)$ & \\
Blunt injury & $(6.0)$ & $(15.0)$ & \\
Drug intoxication; & $(11.4)$ & & \\
Other & & & \\
\hline
\end{tabular}


Table 2. Process measures at the patient-level according to hospital DBD conversion rate,

\begin{tabular}{lccc}
\hline Hospital Population Characteristics & $\begin{array}{c}\text { Low Conversion } \\
\text { (lowest tertile) } \\
\mathrm{n}=464\end{array}$ & $\begin{array}{c}\text { High Conversion } \\
\text { (highest tertile) } \\
\mathrm{n}=100\end{array}$ & $\boldsymbol{p}$ \\
\hline Process measures & & & \\
Consent rate; (\%) & $(52.7)$ & $(85.0)$ & $<0.001$ \\
$\quad$ First-person authorization; (\%) & $(37.8)$ & $(46.1)$ & 0.131 \\
Approached by OPO staff; (\%) & $(55.4)$ & $(71.0)$ & $\mathbf{0 . 0 0 5}$ \\
Timely Referral; (\%) & $(91.8)$ & $(95.0)$ & 0.271 \\
Reasons for declining consent; (\%) & $\mathrm{n}=101$ & $\mathrm{n}=8$ & 0.376 \\
$\quad$ Did not want surgery for their loved one & $(10.0)$ & $(13.3)$ & \\
$\quad$ Did not want to wait & $(10.0)$ & $(20.0)$ & \\
$\quad$ Religion/Cultural & $(13.8)$ & $(0.0)$ & \\
$\quad$ Unaware of loved one's wishes & $(11.0)$ & $(13.3)$ & \\
Previously expressed wishes & $(3.3)$ & $(6.7)$ & \\
Not provided & $(51.9)$ & & \\
\hline
\end{tabular}


Table 3. Hospital characteristics according to conversion rate performance.

\begin{tabular}{lccc}
\hline & $\begin{array}{c}\text { Low Conversion } \\
\text { (lowest tertile) }\end{array}$ & $\begin{array}{c}\text { High Conversion } \\
\text { (highest tertile) }\end{array}$ & $\boldsymbol{p}$ \\
Hospital Characteristics & $\mathrm{n}=17$ & $\mathrm{n}=10$ & $\mathbf{0 . 0 3 4}$ \\
\hline Potential DBD donors volume, median (IQR) & $17(11-32)$ & $7(5-14)$ & $\mathbf{0 . 0 2 4}$ \\
State; (\%) & & & \\
CT & $(41.2)$ & $(0.0)$ & \\
MA & $(52.9)$ & $(60.0)$ & \\
ME & $(0.0)$ & $(10.0)$ & \\
NH & $(0.0)$ & $(20.0)$ & \\
$\quad$ RI & $(5.9)$ & $(10.0)$ & \\
Urbanicity (population $\geq 2.5$ million), (\%) & $(58.8)$ & $(70.0)$ & \\
Ownership; (\%) & & & \\
$\quad$ Private (non-for-profit) & $(82.4)$ & $(80.0)$ & \\
$\quad$ Private (for-profit) & $(17.7)$ & $(20.0)$ & $\mathbf{0 . 0 2 4}$ \\
Bed size, median (IQR) & $337(284-414)$ & $194(166-306)$ & $\mathbf{0 . 0 2 5}$ \\
Teaching hospital, (\%) & $(88.2)$ & $(40.0)$ & $\mathbf{0 . 0 4}$ \\
Trauma center, (\%) & $(76.5)$ & $(30.0)$ & 0.057 \\
Transplant center, (\%) & $(35.3)$ & $(0.0)$ & 0.124 \\
Stroke center, (\%) & $(52.9)$ & $(20.0)$ & $>0.99$ \\
ICU services, (\%) & $(93.3)$ & $(100.0)$ & 0.257 \\
Burn services, (\%) & $(26.7)$ & $(0.0)$ & \\
\hline
\end{tabular}

